selection to produce a distinct animal group. The other finds universally similar processes with similar genes acting to produce an individual animal.

Is it, as the journal's first editorial said, "the birth of a discipline"? Perhaps these two partners are too disparate to provide a unified science. But this attempt to create an intellectual home for the "new synthesis" will make fascinating reading. All the key players are there in the first five issues of the journal. The format is a couple of forum papers and a review, with the rest being mostly original research papers.

Examples of issues raised range from the ancient origins of axial patterning genes through to patterns and rates of vertebral evolution in amphibians and the evolution of the embryonic dorso-ventral axis. Although there is a diverse mix of contributions, spread evenly between the four disciplines, the consistent character is a view based on evolutionary perspectives.

The constraints on the future of this journal will be those of treading a tightrope in the arena of development that will produce an integrated science of biological form. The risk is descent into gene function at the molecular level. The gain would be to achieve the birth of a new platform from which to explain why fossils, with their unimaginable diversity of form, are not just isolated examples of failed experiments in the crucible of life.

http://www.blackwellscience.com/journals/evolution/ index.html

Moya Meredith Smith is in the Department of Craniofacial Development, Dental Institute,

King's College London, University of London, London SE1 9RT, UK.

\section{Crossing the resistance divide}

\section{Drug Resistance Updates}

editor-in-chief Nafsika Georgopapadakou

Churchill Livingstone. 6/yr. \$225, £180

(institutional), \$115, £85 (individual)

\section{Eric J. Arts}

Although resistance is a common and growing concern in the treatment of infectious disease and cancer, Drug Resistance Updates is one of the first journals to address chemotherapeutic resistance as a main theme and to review a wide variety of resistance-related issues.

The bimonthly issues cover a range of topics and pose related questions. For example, what is the mode of resistance to the antimalarial drug chloroquine and should we take this resistance mechanism into account when prescribing newer drugs such

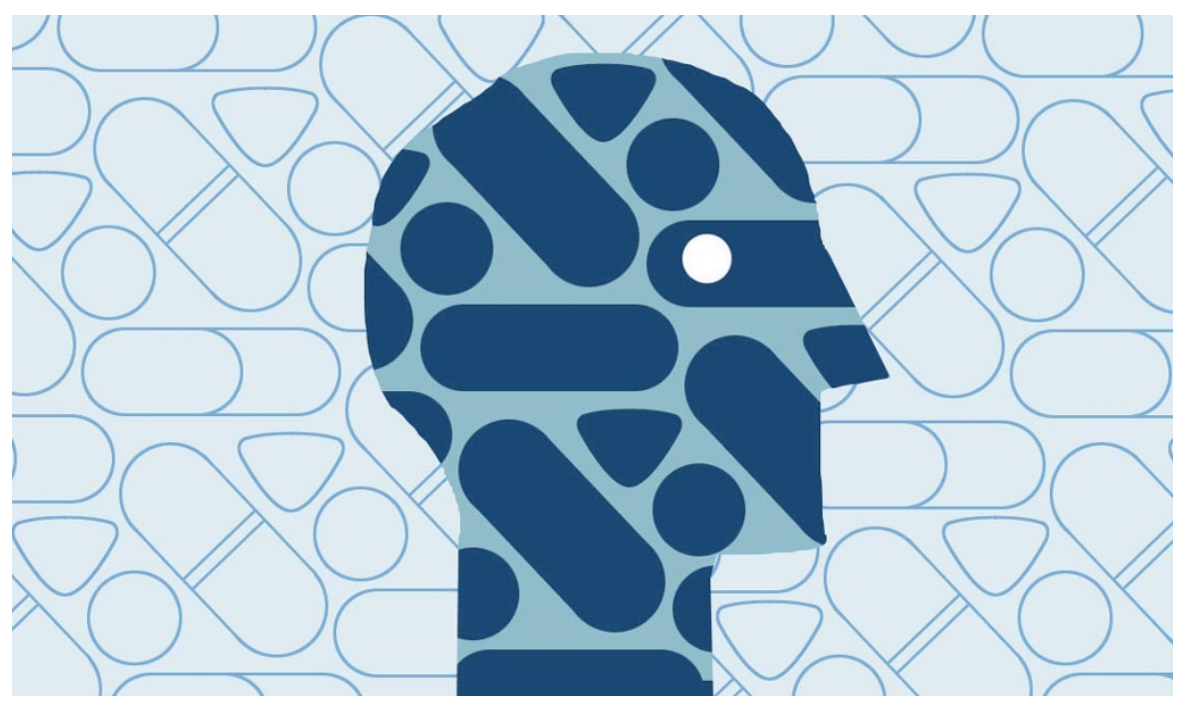

as mefloquine for malaria prophylaxis or treatment? Is the induction of efflux pumps in azole-treated yeast cells analogous to the overexpression of outer membrane proteins acting as efflux pumps in Gram-negative bacteria or that of the MDR-1 P-glycoprotein in human tumour cells?

Similarities between these mechanisms are often difficult to identify because research on drug resistance has been segregated into various reputable but specialized journals focusing on cancer chemotherapy, microbes or viruses. The editors of Drug Resistance Updates have had the good sense to bring together perspectives and reviews on drug resistance in bacteria, cancer, fungi, protozoa and viruses and to provide a simple format for comparing drug-resistance mechanisms between organisms.

The reviews are concise and informative and have recently included descriptive diagrams summarizing complex drugresistance mechanisms. A good mix of established experts and ambitious newcomers as authors provides a fresh perspective on both old and new drug-resistance topics such as bacterial resistance to $\beta$-lactam drugs (for example, penicillin $G$ ) and recent evidence for the resistance of influenza virus to neuraminidase inhibitors (such as zanamivir).

Unlike several other review journals, the areas of microbial and cancer drug resistance are so extensive that there has been little repetition in this journal over its three years of publication. These reasons alone should ensure its survival in a competitive market for high quality and timely reviews.

Although the journal does not solicit comments on previous reviews, it does invite commentaries or perspectives on such topics as managing, characterizing and improving the detection of drug resistance. Even so, more articles comparing drugresistance mechanisms employed by different organisms would add much to this journal's impact. Such comparisons are invaluable for designing new inhibitors, characterizing resistance mechanisms and developing methods to prolong drug use by reversing resistance. In addition to its content and format, the reasonable subscription price justified my purchase of two subscriptions and the use of this journal as a text in our graduate-level course 'Mechanisms of drug resistance'.

\section{http://www.harcourt-international.com/}

\section{journals/drup}

Eric J. Arts is in the Division of Infectious

Diseases, Department of Medicine, Case

Western Reserve University, Cleveland, Ohio 44106, USA.

\title{
Also submitted
}

The following journals were also submitted, but were not reviewed.

- Molecular Cell Biology Research Communications (Academic)

- Physiological Genomics (American Physiological Society)

- Neoplasia (Nature Publishing Group)

- Antioxidants \& Redox Signaling (Mary Ann Liebert)

- Marine Biotechnology (Springer)

- Journal of Alzheimer's Disease (IOS Press)

- Journal of Molecular Microbiology \& Biotechnology (Horizon)

- Systems Engineering (Wiley)

- Cellular Microbiology (Blackwell)

- Cloning (Mary Ann Liebert) 